\title{
Neonatal Maternal Separation Increases Brain-Derived Neurotrophic Factor and Tyrosine Kinase Receptor B Expression in the Descending Pain Modulatory System
}

\author{
Elaine K.Y. Chung ${ }^{a}$ Zhao-Xiang Bian ${ }^{a}$ Hong-Xi Xu ${ }^{b}$ Joseph Jao-Yiu Sung ${ }^{c}$

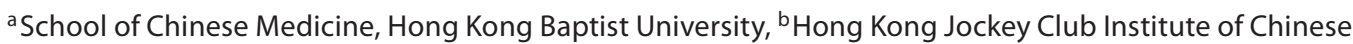 \\ Medicine, and ${ }^{\mathrm{C}}$ Faculty of Medicine, The Chinese University of Hong Kong, Hong Kong, SAR, China
}

\section{Key Words}

Neonatal maternal separation - Colorectal distention • Brain-derived neurotrophic factor $\cdot$ Tyrosine kinase receptor $\mathrm{B} \cdot$ Amygdala $\cdot$ Rostral ventromedial medulla

\begin{abstract}
Neonatal maternal separation (NMS) could trigger longterm changes in the central neuronal responses to nociceptive stimuli in rats. Stress-induced visceral hyperalgesia is closely associated with the dysfunction of descending pain modulatory systems. Brain-derived neurotrophic factor (BDNF) not only has an important role in long-term synaptic plasticity but also in facilitating descending pain. The present study aimed to investigate changes in the expression of $\mathrm{BDNF}$ and its receptor tyrosine kinase receptor $\mathrm{B}$ (TrkB) in the amygdala and the rostral ventromedial medulla (RVM) after NMS and colorectal distention (CRD) stimulation in rats. Male Wistar rat pups were subjected to $180 \mathrm{~min}$ of daily NMS or not handled for 13 consecutive days. Expression of BDNF and TrkB following NMS and CRD stimulation was determined using immunohistochemistry. The results revealed an increase in the expression of BDNF and TrkB in the amygdala after NMS. An interactive effect of NMS and CRD on the expression of TrkB, but not BDNF, was found in the RVM. Furthermore, a significant interactive effect of NMS and CRD on the colocalization coefficient of TrkB and phospho-extracel-
\end{abstract}

lular signal-regulated kinase expression in both the amygdala and RVM were found. These data demonstrate that NMS increases BDNF and TrkB expression in the descending pain systems, which may contribute to the development of NMSinduced visceral hyperalgesia.

Copyright $\odot 2009$ S. Karger AG, Basel

\section{Introduction}

Neonatal maternal separation (NMS) from postnatal days 2 to 14 has been shown to have a long-lasting influence on the development of the neural systems of rats [1-3]. In previous studies, we found that NMS rats developed visceral hyperalgesia and enhanced neuronal sensitivity to noxious visceral stimuli in the central brain nuclei $[4,5]$. These nuclei, including the cingulate cortex, the thalamus and the amygdala, play important roles in the central pain matrix and are involved in generating emotional and autonomic responses $[6,7]$.

The amygdala, in particular, plays a central role in the emotional affective component of pain [8-10]. It has important roles in descending pain enhancement and inhibition control of the interneurons in the spinal cord through the relay site of the rostral ventromedial medulla (RVM) $[11,12]$. Neuronal plasticity changes in the amygdala have been found to be involved in the genera-

Dr. Bian Zhao-Xiang

School of Chinese Medicine

Hong Kong Baptist University

Kowloon Tong, Hong Kong, SAR (China)

Tel. +852 3411 2929, Fax +852 3411 2905, E-Mail bzxiang@hkbu.edu.hk 
tion and maintenance of central sensitization in animals and humans $[8,13,14]$. Strong evidence for long-term potentiation in the amygdala has been found in various pain models [15-19]. The extracellular signal-regulated kinases (Erks) have been identified as molecular substrates underlying this long-term potentiation plasticity change in the amygdala in an inflammation-induced pain model $[20,21]$. The RVM forms the primary relay site and exerts a biphasic influence on visceral nociceptive transmission $[22,23]$. The increased excitability in response to visceral stimuli in the spinal cord after NMS, observed in our previous studies [4, 24], reflects changes in plasticity in descending pain circuitry. Our recent work has shown differential regulation of phospho (p)Erk and c-Fos expression in the central pain matrix [5]. However, the mechanisms underlying these changes in plasticity are unknown.

Brain-derived neurotrophic factor (BDNF) has been shown to contribute to the synaptic plasticity formation in learning and memory [25-27] as well as the long-term plasticity changes in the central pain matrix [28]. A previous study found that BDNF expression was downregulated in the hippocampus and the striatum but upregulated in the ventral tegmental area of adult rats following postnatal maternal separation [29]. A growing body of evidence has indicated that supraspinal BDNF signaling is a novel descending pain facilitation pathway in nociceptive processing in the spinal cord [30]. These findings suggest that BDNF is an endogenous stress-responsive mediator in the brain. Therefore, we hypothesize that changes in the expression of BDNF and its receptor tyrosine kinase receptor B (TrkB) in descending pain circuitry may contribute to the development of stress-induced visceral hyperalgesia. The present study aimed to map the changes in BDNF signaling in the amygdala and RVM in an animal model of NMS-induced visceral hyperalgesia.

\section{Materials and Methods}

\section{Animals}

Primiparous timed-pregnant female Wistar rats were obtained from the Laboratory Animal Services Center, The Chinese University of Hong Kong. Dams were housed individually in plastic cages and maintained on a 12-hour light/dark cycle with free access to food and water. The handling of rats and all procedures performed were approved in accordance with the Animals (Control of Experiments) Ordinance, Hong Kong, China.

\section{Maternal Separation}

On postnatal days 2-14, pups were moved from their maternity cages to adjacent cages of an identical type for $180 \mathrm{~min}$ daily, as described previously [31]. After the separation period, pups were returned to their maternity cages. Control groups were not exposed to handling [nonhandled $(\mathrm{NH})$ rats] and were maintained in their maternity cages with the dams. On postnatal day 22 , the sex of the pups was determined. Female pups were culled, and male pups were weaned and housed in individual cages. After weaning, pups were weighed weekly. Only male rats weighing 250-300 g were used in the present study.

\section{Colorectal Distention}

One group each of NMS rats and NH rats ( $\mathrm{n}=6$ for each group) was randomly chosen for the colorectal distention (CRD) experiments. A series of twenty 20 -second distensions of $80 \mathrm{~mm} \mathrm{Hg}$ was performed, as previously described [32]. Intervals between balloon stimulations were $4 \mathrm{~min}$. One hour after the final stimulation, rats were deeply anesthetized with an overdose of midazolam hydrochloride and transcardially perfused. The remaining groups of NMS and $\mathrm{NH}$ rats ( $\mathrm{n}=3$ for each group) were lightly anesthetized with ether, without CRD stimulus, and then deeply anesthetized and perfused.

\section{Immunohistochemistry}

Tissue Preparation. The brain and the lumbar-sacral spinal cord segments were removed and postfixed in fixative overnight, postfixed in $30 \%$ sucrose in $0.1 \mathrm{M} \mathrm{PBS}$ at $4^{\circ} \mathrm{C}$ for approximately 3 days and then stored at $-80^{\circ} \mathrm{C}$ with embedding matrix (Shandon Cryomatrix, Thermo Electron Corporation, Waltham, Mass., USA). Coronal sections $(30 \mu \mathrm{m})$ were cut on a freezing cryostat.

Single Immunohistochemistry Staining. Immunohistochemistry was performed as previously described [24]. Sections were blocked with $20 \%$ normal horse serum in PBS for $1 \mathrm{~h}$ followed by incubation with rabbit polyclonal antibody solutions against BDNF (1:1,000 in $0.3 \%$ Triton X-100 containing $0.02 \%$ sodium azide in $2 \times$ PBS; Chemicon International, Billerica, Mass., USA) or TrkB (1:2,000 in $0.3 \%$ Triton X-100 in PBS; Chemicon International) for $48 \mathrm{~h}$ at $4^{\circ} \mathrm{C}$. Following primary antibody incubation, the sections were rinsed 3 times for 5 min each in PBS and then incubated for $2 \mathrm{~h}$ with a biotinylated secondary antibody (1:200 in PBS; Vector Laboratories, Burlingame, Calif., USA) at room temperature. Following incubation in a solution containing avidin-biotin complex (Elite ABC kit, Vector Laboratories) for 45 min at room temperature and subsequent reaction with diaminobenzidine, sections were mounted on gelatin-coated slides, dehydrated in a series of graded alcohol and coverslipped.

Double Immunofluorescence Staining. Sections were incubated in a mixture of mouse monoclonal antibody against $\mathrm{p}$-Erk1/2 (1:300; Cell Signaling Technology) with either rabbit polyclonal antibody against BDNF (1:1,000; Chemicon International) or TrkB (1:2,000; Chemicon International) in PBS containing $0.3 \%$ Triton X-100 for $48 \mathrm{~h}$ at $4^{\circ} \mathrm{C}$. Following primary antibody incubation, sections were rinsed 3 times in PBS and then incubated for $2 \mathrm{~h}$ in a secondary antibody cocktail consisting of Alexa Fluor 488conjugated secondary antibodies and Alexa Fluor 568-conjugated secondary antibodies (1:500; Molecular Probes, Eugene, Oreg., USA). After the final incubation, sections were rinsed 3 times in PBS and then coverslipped with mounting medium (Vectashield, Vector Laboratories). Finally, sections were imaged with a laser scanning microscope (Olympus Fluoview FV1000). The absence of cross-reactivity was confirmed by a single-labeled control preparation. 
Fig. 1. Immunoreactivity for BDNF is detected in the neuropil of the CeA of $\mathrm{NH}(\mathbf{A}$, B) and NMS (C, D) rats with or without CRD. Arrows indicate intense staining of BDNF in close proximity to cell-like structures (A-D). Higher expression of BDNF is observed in the NMS rats $(\mathbf{C}, \mathbf{D})$ compared to the $\mathrm{NH}$ rats $(\mathbf{A}, \mathbf{B})$. Scale bar $=500 \mu \mathrm{m}$ (A-D).
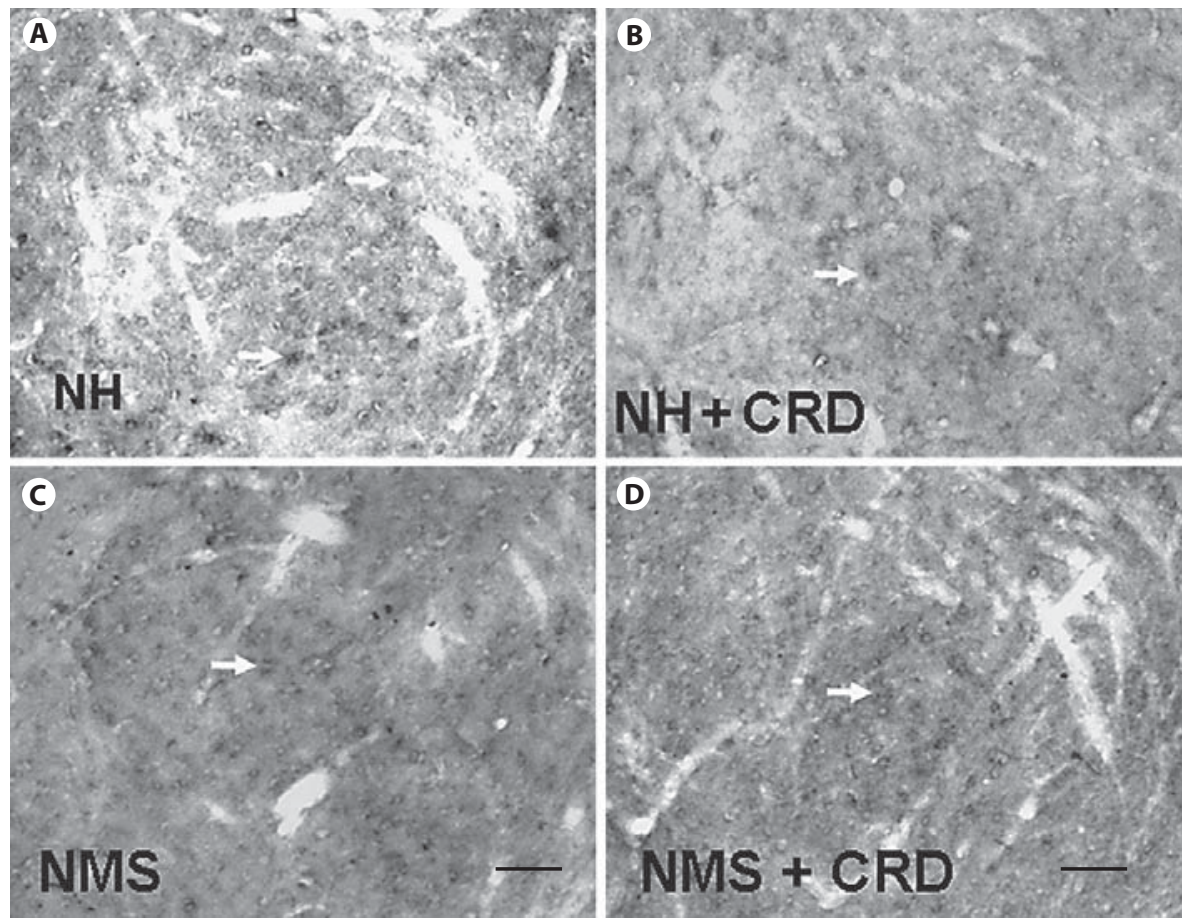

Controls. Controls for the single and double immunohistochemistry trials were performed by omission of primary antibodies in original reaction sequences, as described in previous studies [4].

\section{Densitometry Measurement}

To measure the level of intensity of BDNF and TrkB immunoreactivity, digital images of the sections were obtained using a light microscope (Axiovert 200, Zeiss) under standard illumination conditions. Images at $200 \times$ magnification were then analyzed by an image analysis program (Image J, NIH). Measurements of the calibrated optical densities indicating the levels of BDNF and TrkB immunoreactivity in the amygdala and the RVM were determined. The data from at least 3 sections from each animal were averaged and expressed as the mean \pm standard error of the mean (SEM).

To determine the percentage of colocalization of $\mathrm{p}$-Erk $1 / 2$ and either BDNF or TrkB immunoreactivity, images from double-immunostained sections were captured under the same parameters in the confocal microscope (Fluoview FV1000, Olympus) at high magnification (using a $60 \times$ lens). The pinhole was set to 1 Airy unit, and sections were scanned 3 times to reduce noise. Images acquired at a digital size of $1,024 \times 1,024$ were used to determine the percentage of colocalization using image analyzing software (Image J, NIH). The 'image correlator plus' plugin of Image J was used to generate the Pearson's colocalization coefficient. This was done by measuring the overlap of pixels from 2 channels of each image. Then the coefficients from each group were averaged and expressed as the mean \pm SEM.

Statistical comparisons between groups were conducted using two-way ANOVA with repeated measures followed by a post hoc test with Bonferroni correction.

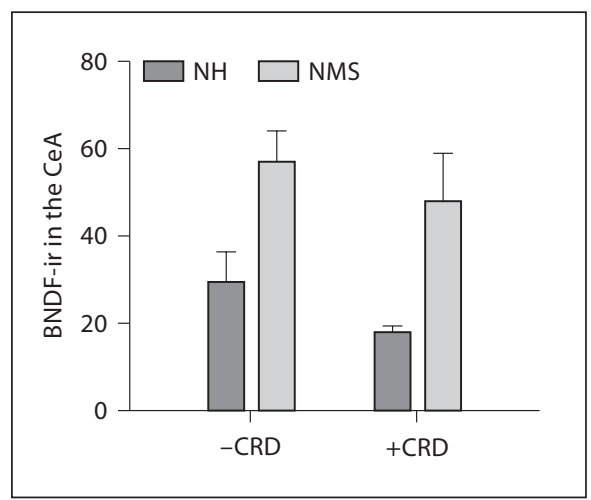

Fig. 2. A significant NMS effect on the expression of BDNF in the CeA was found ( $\mathrm{n}=3 ; \mathrm{p}=0.002, \mathrm{~F}=18.18$; two-way ANOVA). Bars represent the mean \pm SEM of the average optical density of the immunoreactivity for BDNF (BDNF-ir).

\section{Results}

\section{Upregulation of BDNF and TrkB Expression in the Central Nuclei of the Amygdala after NMS}

BDNF immunoreactivity was seen in the neuropil of the amygdala (fig. 1). The neuronal cell bodies were clearly stained in each experimental group of rats (fig. 1A-D). Semiquantitative analyses of the density of immunoreac- 
Fig. 3. TrkB-immunoreactive perikarya and neuropil are shown in the CeA of $\mathrm{NH}$ (A, B) and NMS (C, D) rats with or without CRD. More intense staining of TrkB is observed in NMS rats $(\mathbf{C}, \mathbf{D})$ than in $\mathrm{NH}$ rats (A, B). Scale bar $=500 \mu \mathrm{m}(\mathbf{A}-\mathbf{D})$.
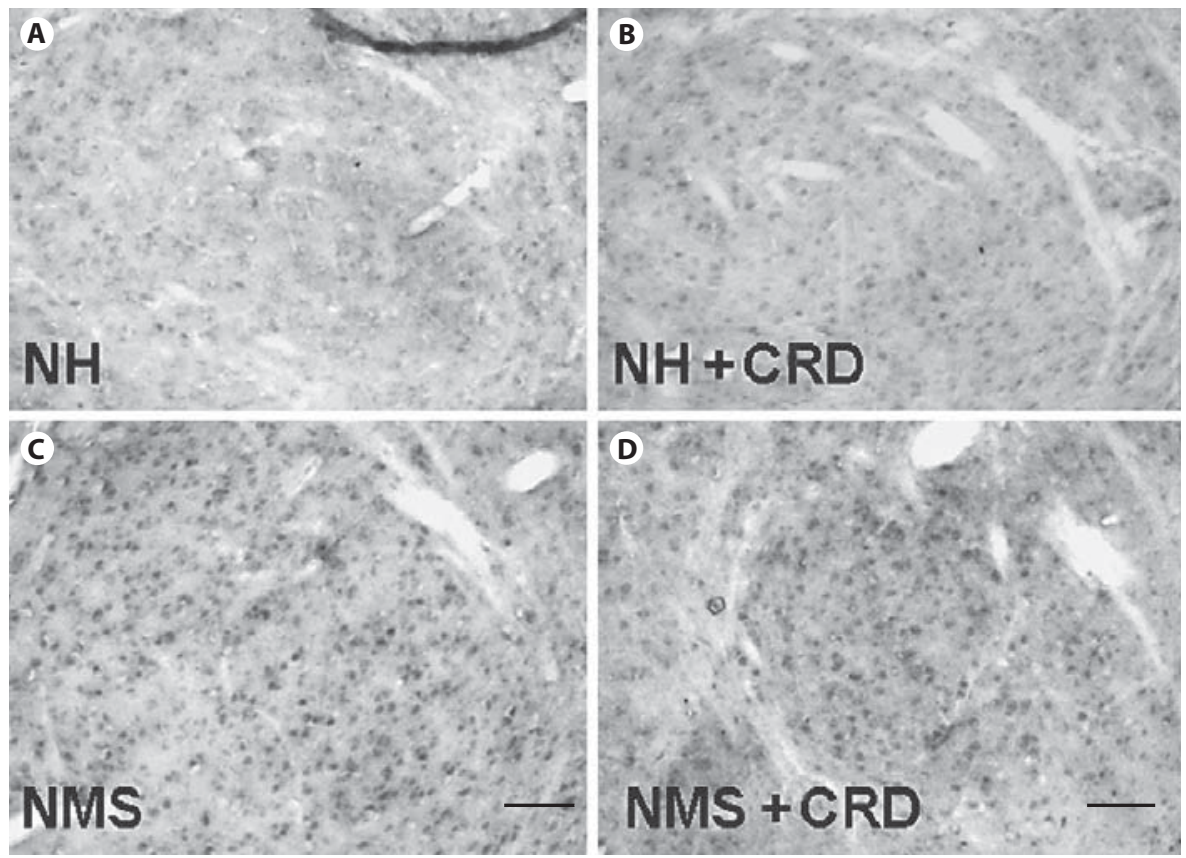

tivity for BDNF in a fixed area within the central nuclei of the amygdala $(\mathrm{CeA})$ revealed a significant effect of NMS on the level of expression of BDNF ( $p=0.002, F=$ $18.18 ; \mathrm{n}=3)$. Upregulation of BDNF expression in the CeA was found after NMS (fig. 2).

A similar pattern was found for $\operatorname{TrkB}$ expression. TrkB-positive cell bodies were observed in the CeA of each group of rats (fig. 3A-D). Semiquantitative analyses revealed a significant effect of NMS, but not CRD, on the expression of TrkB in the CeA ( $\mathrm{p}=0.037, \mathrm{~F}=5.98 ; \mathrm{n}=3)$. Upregulation of TrkB expression was found after NMS (fig. 4).

\section{Interactive Effect of NMS and CRD on TrkB \\ Expression in $R V M$}

In the RVM, TrkB immunoreactivity was observed in the neurons (fig. 5A-D). TrkB expression increased significantly after CRD stimulation in NMS rats (fig. 6). Semiquantitative analyses of the density of TrkB immunoreactivity indicate that there was a significant interactive effect of NMS and CRD on the expression of TrkB in the RVM ( $\mathrm{p}=0.006, \mathrm{~F}=12.192 ; \mathrm{n}=3)$.

Increased Colocalization of TrkB on p-Erk1/2-Positive

Neurons in the CeA and RVM in NMS Rats

Double immunofluorescence revealed staining for $\mathrm{p}$ Erk1/2 and TrkB localized in the same neuronal perikarya in the CeA (fig. 7). A strong correlation between

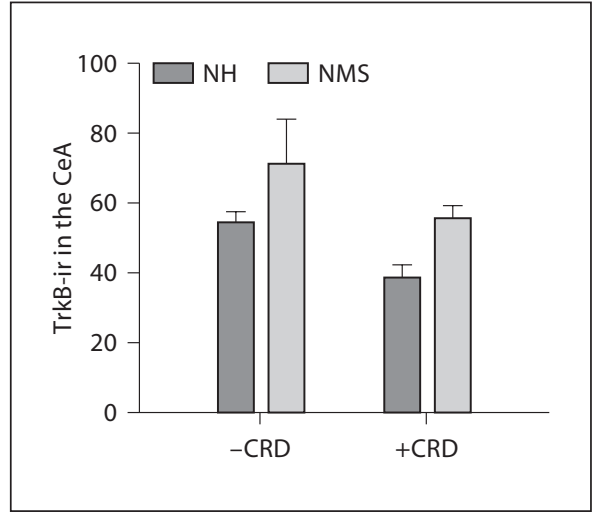

Fig. 4. A significant NMS effect on the expression of TrkB in the CeA was found ( $\mathrm{n}=3 ; \mathrm{p}=0.037, \mathrm{~F}=5.979$; two-way ANOVA). Bars represent the mean \pm SEM of the average optical density of the immunoreactivity for TrkB (TrkB-ir).

p-Erk1/2 and TrkB expression was found in all groups of rats (fig. 7A-D). Analyses of the colocalization coefficient of the 2 immunoreactive products revealed a significant interactive effect of NMS and CRD on the proportion of colocalization of TrkB and $\mathrm{p}-\mathrm{Erk} 1 / 2$ in the CeA $(\mathrm{p}=$ $0.041, \mathrm{~F}=4.77 ; \mathrm{n}=6$ ). Under CRD stimulation, a higher colocalization coefficient of TrkB on p-Erk1/2-positive neurons was found in NMS rats as compared to $\mathrm{NH}$ rats (fig. 8). 
Fig. 5. Strong labeling of TrkB immunoreactivity in the perikarya in the RVM of $\mathrm{NH}(\mathbf{A}, \mathbf{B})$ and NMS (C, D) rats with or without CRD. Upregulation of TrkB immunoreactivity is observed in NMS + CRD rats (D). Scale bar $=500 \mu \mathrm{m}(\mathbf{A}-\mathbf{D})$.
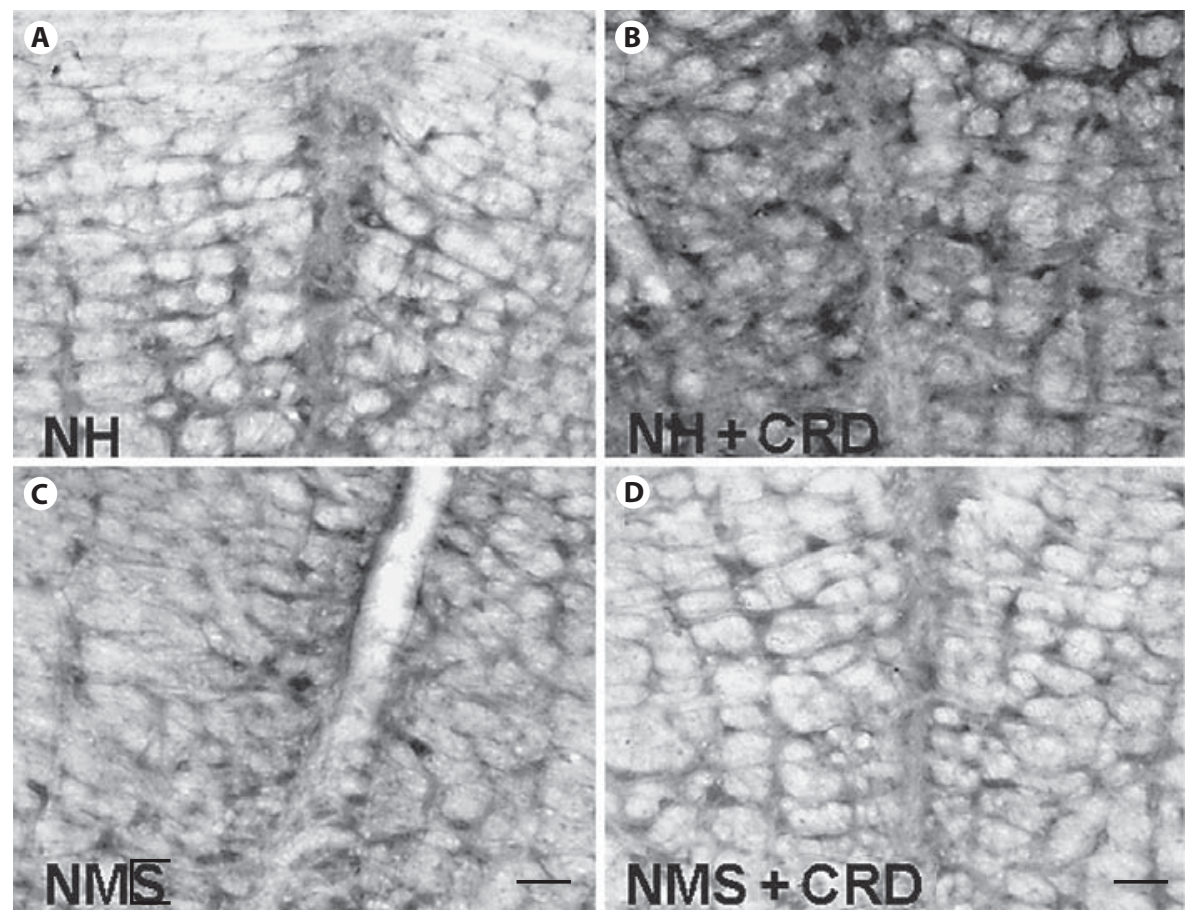

In the RVM, TrkB- and p-Erk1/2-positive neurons were observed (fig. 9). A significant interactive effect of NMS and CRD on the proportion of colocalization of TrkB and $\mathrm{p}-$ Erk1/2 was found $(\mathrm{p}=0.01, \mathrm{~F}=8.16 ; \mathrm{n}=6)$. Under CRD stimulation, the proportion of TrkB expression in p-Erk1/2-positive neurons was found to be higher in NMS rats as compared to NH rats (fig. 10).

\section{Discussion}

In the present study, BDNF and TrkB expression was upregulated in the CeA after NMS. An interactive effect of NMS and CRD on the expression of TrkB in the RVM was found, which was demonstrated by an upregulation of TrkB expression in the NMS rats that underwent CRD stimulation. Double immunostaining revealed a significant interactive effect of NMS and CRD on the proportion of colocalization of TrkB with p-Erk1/2 in both the $\mathrm{CeA}$ and RVM. In summary, the present results provide evidence of changes in BDNF and TrkB expression within the descending pain circuitry, and these changes may play an important role in the development of visceral hyperalgesia in NMS rats.

A growing body of evidence has suggested that stressinduced neuronal plasticity changes in the brain are as-

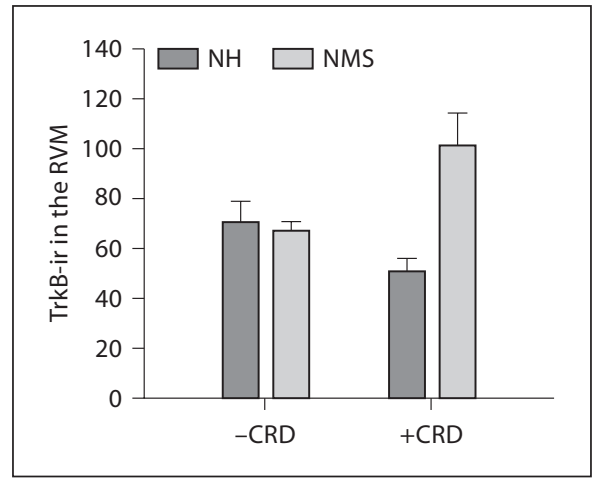

Fig. 6. A significant interactive effect of NMS and CRD on the expression of TrkB in the RVM was found $(n=3 ; p=0.006, F=$ 12.19; two-way ANOVA). Bars represent the mean \pm SEM of the average optical density of the immunoreactivity for TrkB (TrkBir).

sociated with modulations in BDNF signaling. Psychological stresses increase BDNF expression in the hippocampus, striatum and ventral tegmental area in rats [29, 33], and chronic stress applied to adult rats causes upregulation of $\mathrm{BDNF}$ in the amygdala $[34,35]$. These data point to the fact that BDNF is an important endogenous mediator of stress responses within the brain. The cur- 
$\mathrm{NH}$
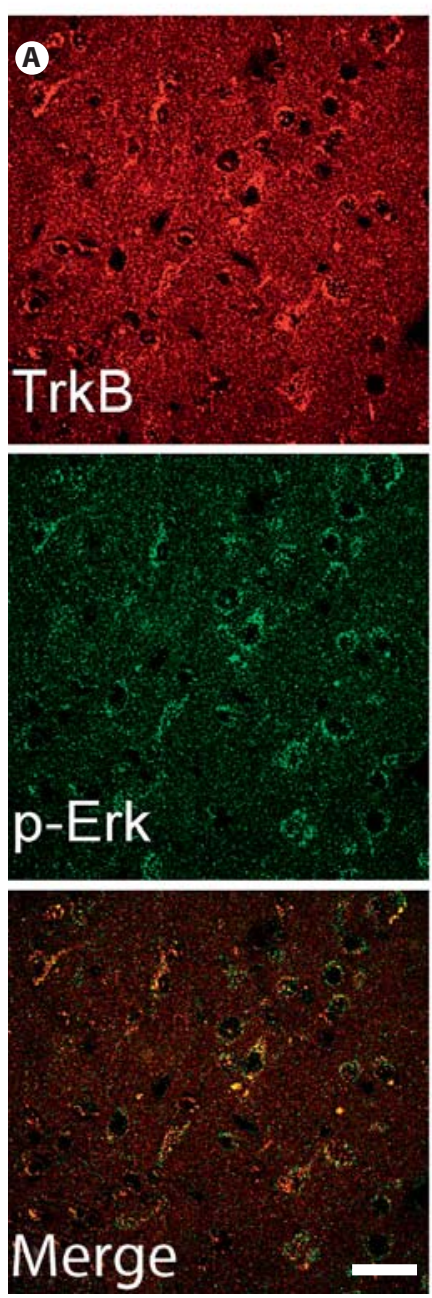

NMS
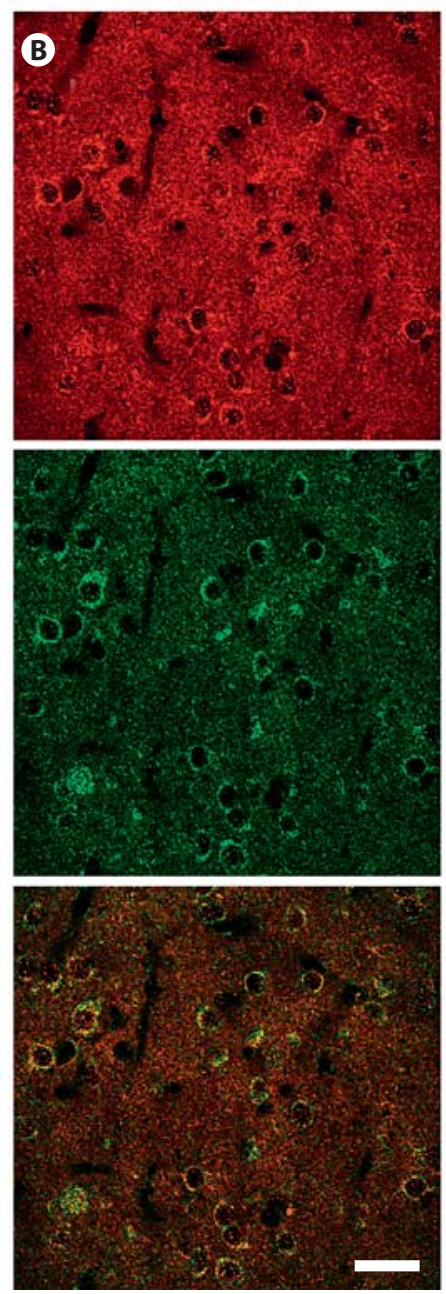

$\mathrm{NH}+\mathrm{CRD}$
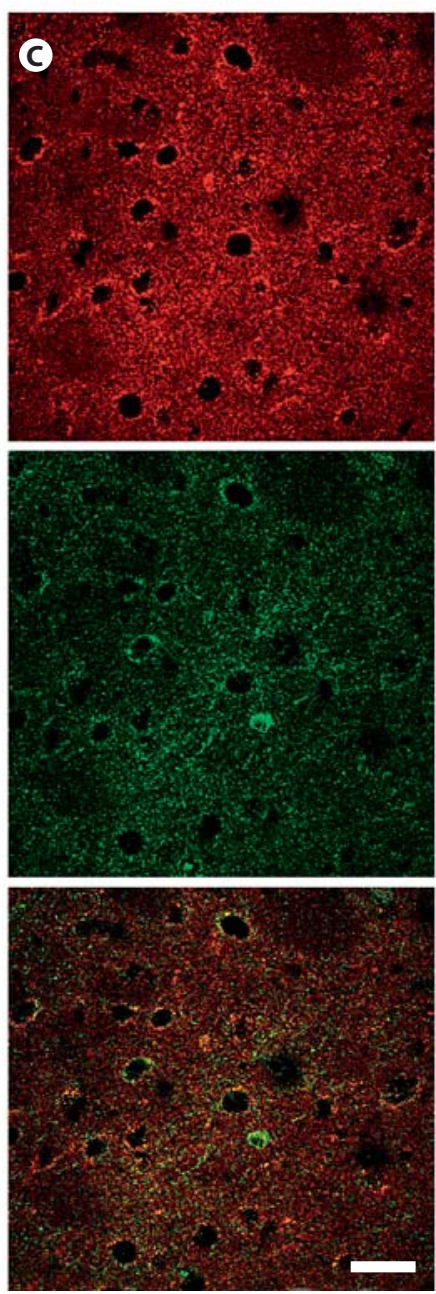

NMS + CRD
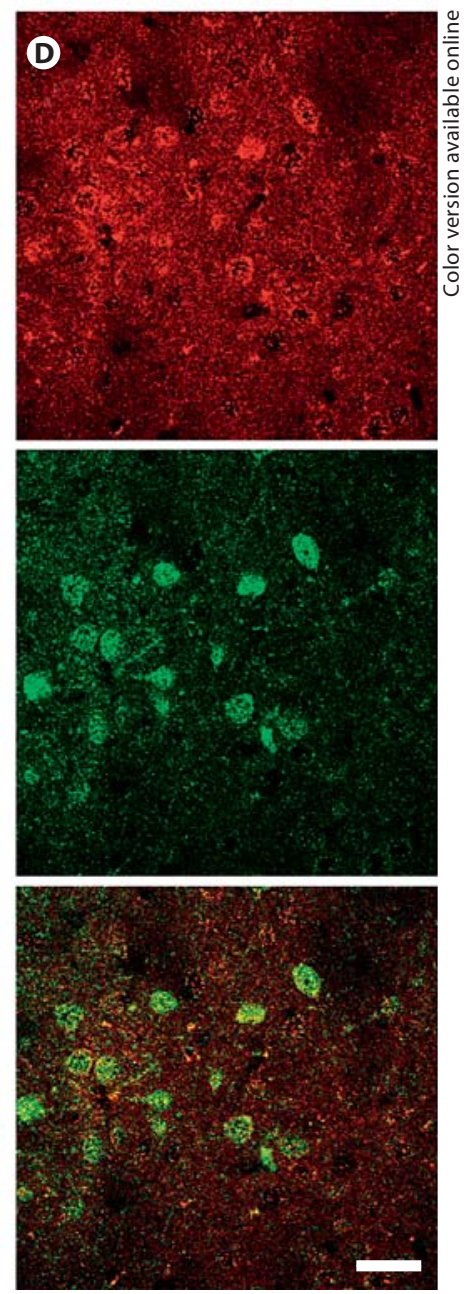

Fig. 7. Fluorescent micrographs of the amygdala of rats double immunostained to reveal immunoreactivity for TrkB and p-Erk. TrkB and p-Erk colocalize on the same neurons, as indicated by the overlapping signals (merge) which appear prominently in NH (A), NMS (B), NH + CRD (C) and NMS + CRD (D) rats. Scale bar $=200 \mu \mathrm{m}$.

Fig. 8. A significant interactive effect of NMS and CRD on the expression of TrkB in $\mathrm{p}$-Erk-positive neurons was found $(\mathrm{n}=$ $6 ; \mathrm{p}=0.041, \mathrm{~F}=4.772$; two-way ANOVA). Bars represent the mean \pm SEM of the colocalization coefficient for TrkB and p-Erk immunoreactivity (TrkB-ir; p-Erk1/2-ir) in the CeA.

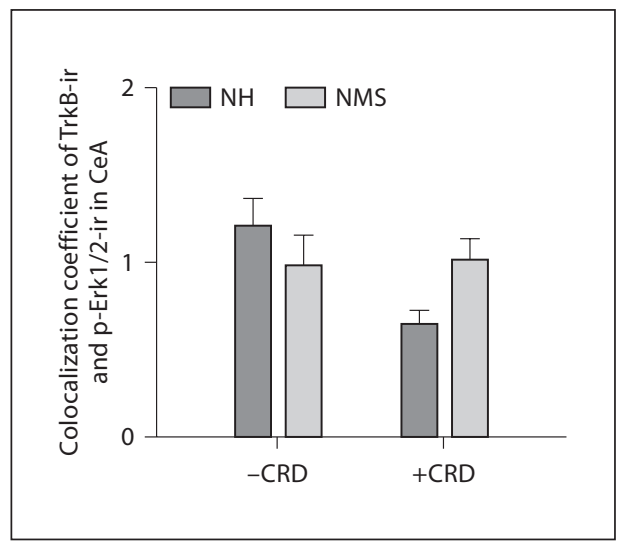



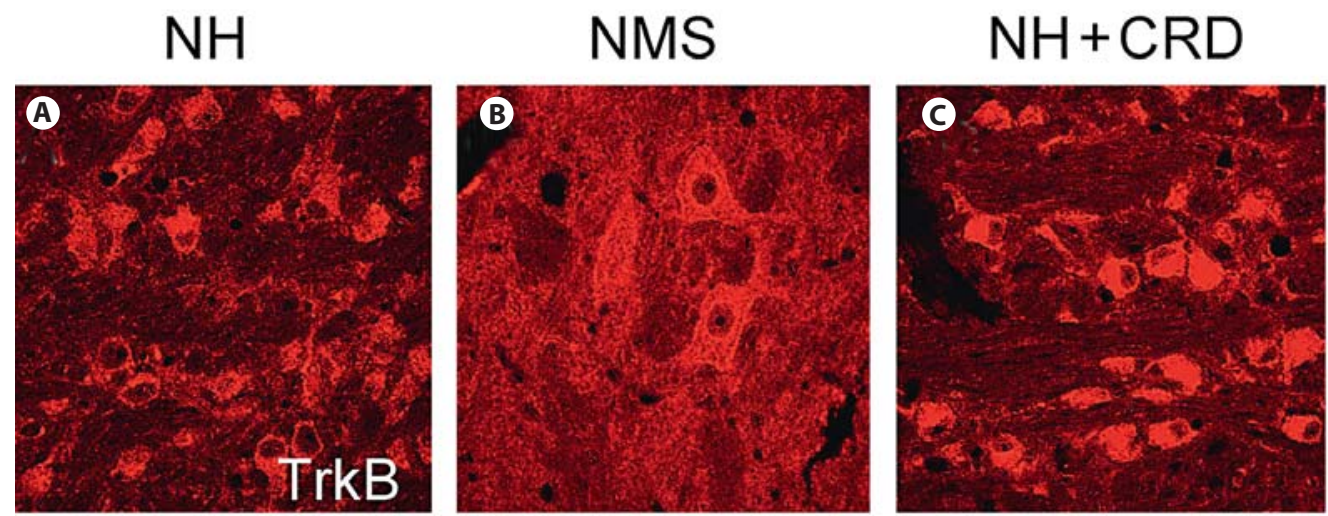

$\mathrm{NMS}+\mathrm{CRD}$
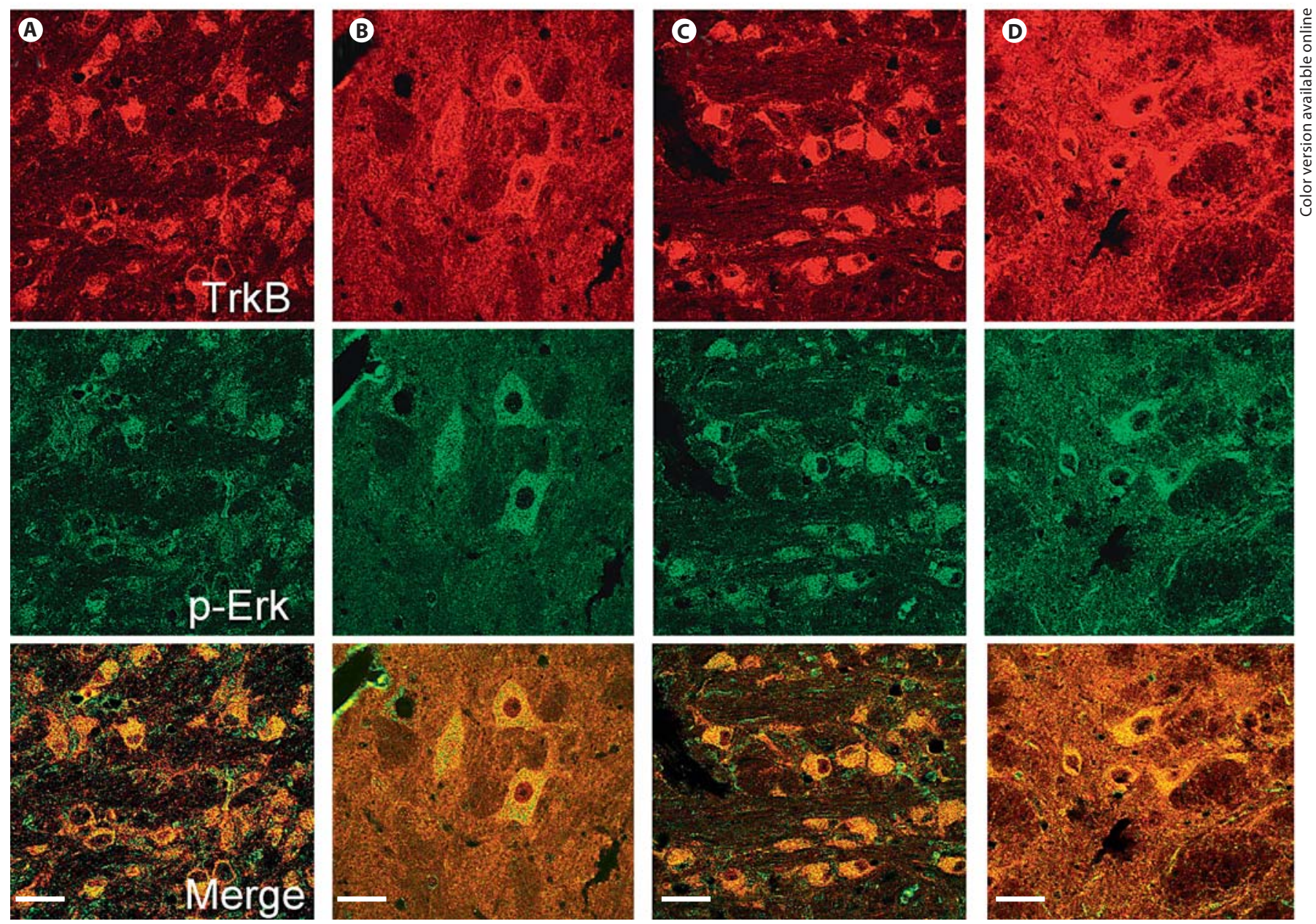

Fig. 9. Fluorescent micrographs of the RVM of rats double immunostained to reveal immunoreactivity for TrkB and p-Erk. TrKB and p-Erk colocalize, as indicated by the overlapping signals (Merge) which appear prominently in NH (A), NMS (B), NH + CRD (C) and NMS + CRD (D) rats. Scale bar $=200 \mu \mathrm{m}$.

Fig. 10. A significant interactive effect of NMS and CRD on the colocalization coefficient of TrkB and p-Erk immunoreactivity (TrkB-ir; p-Erk1/2-ir) in the perikarya in the RVM was found $(\mathrm{n}=6 ; \mathrm{p}=0.01, \mathrm{~F}=8.162$; two-way ANOVA). Bars represent the mean \pm SEM of the colocalization coefficient for TrkB and p-Erk.

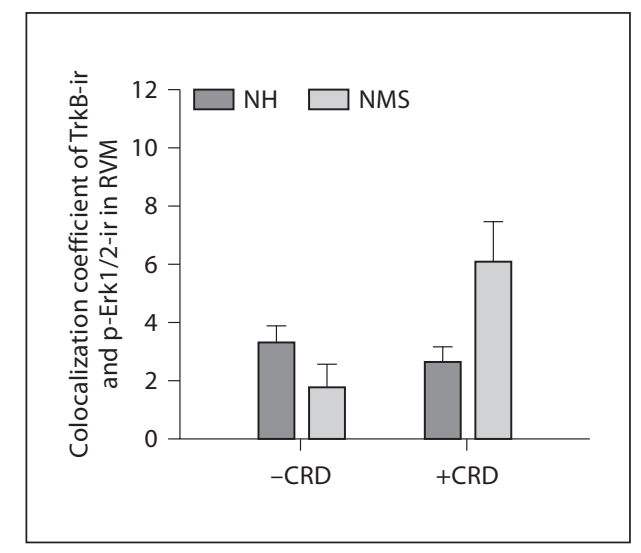


rent data clearly demonstrate that BDNF and TrkB expression is upregulated in the amygdala after NMS. BDNF-induced synaptic plasticity, such as long-term potentiation in the amygdala, is mediated by Erks and plays a central role in the learning of fear [21]. Activation of Erk signaling in the amygdala was found to be necessary to induce long-lasting tactile hypersensitivity in an inflammation-induced pain model [20]. These changes in the amygdala may contribute to the increase in neuronal activity in NMS-induced visceral hyperalgesia.

Our recent study showed that p-Erk expression increased in the amygdala in NMS rats [5]. The present results provide evidence for the colocalization of p-Erkpositive neurons and TrkB immunoreactivity. An analysis of the colocalization coefficient revealed that the proportion of p-Erk-positive neurons displaying TrkB immunoreactivity was significantly increased in the amygdala under the interactive effect of NMS and CRD. These findings suggest that BDNF signaling may be involved in the plasticity change in the amygdala that contributes to the NMS-induced visceral hyperalgesia. Given the well-established role of the amygdala in both emotional responses and pain processing, it is possible that neuromodification in the amygdala is involved in central hyperalgesia in NMS rats.

A previous study demonstrated that the nuclei of the amygdala are involved in the RVM nociceptive modulatory systems [12]. Environmental stimuli likely trigger modifications in the descending pain modulatory sys- tems through the neuronal circuits linking the amygdala and RVM. Enhanced activation of TrkB receptors in the RVM was previously found to contribute to descending facilitation of inflammatory pain [30]. Abundant behavioral pharmacological studies have demonstrated the functional role of TrkB in RVM neurons in pain facilitation through the effect of intra-RVM injection of antiBDNF antiserum or TrkB-IgG fusion protein in attenuating central hyperalgesia. In the present study, we demonstrated a significant increase in $\operatorname{TrkB}$ expression in the $\mathrm{RVM}$, the relay site between the amygdala and the spinal cord, under the joint influence of NMS and CRD. Study of the colocalization coefficient also revealed a significant interactive effect of NMS and CRD on the proportion of p-Erk-positive neurons expressing TrkB immunoreactivity. These data provide evidence for the modification of BDNF and TrkB in the amygdala-RVM circuit and neuronal response to CRD in NMS rats. These findings could serve as a basis for further investigations on the pharmacological and functional roles of BDNF in synaptic activity related to pain modulation in NMS-induced visceral hyperalgesia.

\section{Acknowledgements}

The present work was supported by the Hong Kong Jockey Club Institute of Chinese Medicine (JCICM-4-07) and the Research Grants Council, Hong Kong (HKBU 260008).

\section{References}

1 Liu D, Caldji C, Sharma S, Plotsky PM, Meaney MJ: Influence of neonatal rearing conditions on stress-induced adrenocorticotropin responses and norepinepherine release in the hypothalamic paraventricular nucleus. J Neuroendocrinol 2000;12:5-12.

$\checkmark 2$ Caldji C, Tannenbaum B, Sharma S, Francis D, Plotsky PM, Meaney MJ: Maternal care during infancy regulates the development of neural systems mediating the expression of fearfulness in the rat. Proc Natl Acad Sci USA 1998; $95: 5335-5340$.

3 Coutinho SV, Plotsky PM, Sablad M, Miller JC, Zhou H, Bayati AI, McRoberts JA, Mayer EA: Neonatal maternal separation alters stress-induced responses to viscerosomatic nociceptive stimuli in rat. Am J Physiol Gastrointest Liver Physiol 2002;282:G307G316.

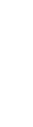

$\checkmark 8$ Neugebauer V: The amygdala: different pains, different mechanisms. Pain 2007;127: $1-2$. hances central sensitivity to noxious colorectal distention in rat. Brain Res 2007;1153: 68-77.

5 Zhang XJ, Li Z, Chung EK, Zhang HQ, Xu HX, Sung JJ, Bian ZX: Activation of extracellular signal-regulated protein kinase is associated with colorectal distension-induced spinal and supraspinal neuronal response and neonatal maternal separation-induced visceral hyperalgesia in rats. J Mol Neurosci 2008;37:274-287.

6 Jones MP, Dilley JB, Drossman D, Crowell MD: Brain-gut connections in functional GI disorders: anatomic and physiologic relationships. Neurogastroenterol Motil 2006; 18:91-103.

7 Mayer EA: Spinal and supraspinal modulation of visceral sensation. Gut 2000;47(suppl 4):iv69-iv72.
79 Phelps EA, LeDoux JE: Contributions of the amygdala to emotion processing: from animal models to human behavior. Neuron 2005;48:175-187.

10 Kalin NH, Shelton SE, Davidson RJ: The role of the central nucleus of the amygdala in mediating fear and anxiety in the primate. J Neurosci 2004;24:5506-5515.

11 Fields HL: Pain modulation: expectation, opioid analgesia and virtual pain. Prog Brain Res 2000;122:245-253.

12 McGaraughty S, Heinricher MM: Microinjection of morphine into various amygdaloid nuclei differentially affects nociceptive responsiveness and RVM neuronal activity. Pain 2002;96:153-162. 
13 Neugebauer V, Li W, Bird GC, Han JS: The amygdala and persistent pain. Neuroscientist 2004;10:221-234.

14 Rosen JB, Schulkin J: From normal fear to pathological anxiety. Psychol Rev 1998;105: 325-350.

15 Han JS, Li W, Neugebauer V: Critical role of calcitonin gene-related peptide 1 receptors in the amygdala in synaptic plasticity and pain behavior. J Neurosci 2005;25:1071710728.

-16 Han JS, Neugebauer V: mGluR1 and mGluR5 antagonists in the amygdala inhibit different components of audible and ultrasonic vocalizations in a model of arthritic pain. Pain 2005; 113:211-222.

- 17 Pedersen LH, Scheel-Kruger J, BlackburnMunro G: Amygdala GABA-A receptor involvement in mediating sensory-discriminative and affective-motivational pain responses in a rat model of peripheral nerve injury. Pain 2007;127:17-26.

-18 Tanimoto S, Nakagawa T, Yamauchi Y, Minami M, Satoh M: Differential contributions of the basolateral and central nuclei of the amygdala in the negative affective component of chemical somatic and visceral pains in rats. Eur J Neurosci 2003;18:23432350 .

19 Ikeda R, Takahashi Y, Inoue K, Kato F: NMDA receptor-independent synaptic plasticity in the central amygdala in the rat model of neuropathic pain. Pain 2007;127:161172 .

20 Carrasquillo Y, Gereau RW: Activation of the extracellular signal-regulated kinase in the amygdala modulates pain perception. J Neurosci 2007;27:1543-1551.
21 Ou LC, Gean PW: Regulation of amygdaladependent learning by brain-derived neurotrophic factor is mediated by extracellular signal-regulated kinase and phosphatidylinositol-3-kinase. Neuropsychopharmacology 2006;31:287-296.

22 Zhuo M, Sengupta JN, Gebhart GF: Biphasic modulation of spinal visceral nociceptive transmission from the rostroventral medial medulla in the rat. J Neurophysiol 2002;87: 2225-2236.

23 Zhuo M, Gebhart GF: Facilitation and attenuation of a visceral nociceptive reflex from the rostroventral medulla in the rat. Gastroenterology 2002;122:1007-1019.

24 Chung EK, Zhang XJ, Xu HX, Sung JJ, Bian ZX: Visceral hyperalgesia induced by neonatal maternal separation is associated with nerve growth factor-mediated central neuronal plasticity in rat spinal cord. Neuroscience 2007;149:685-695.

25 Bekinschtein P, Cammarota M, Izquierdo I, Medina JH: BDNF and memory formation and storage. Neuroscientist 2008;14:147156.

26 Figurov A, Pozzo-Miller LD, Olafsson P, Wang T, Lu B: Regulation of synaptic responses to high-frequency stimulation and LTP by neurotrophins in the hippocampus. Nature 1996;381:706-709.

27 Lu B, Chow A: Neurotrophins and hippocampal synaptic transmission and plasticity. J Neurosci Res 1999;58:76-87.

28 Ren K, Dubner R: Pain facilitation and activity-dependent plasticity in pain modulatory circuitry: role of BDNF-TrkB signaling and NMDA receptors. Mol Neurobiol 2007;35: 224-235.

-29 Lippmann M, Bress A, Nemeroff CB, Plotsky PM, Monteggia LM: Long-term behavioural and molecular alterations associated with maternal separation in rats. Eur J Neurosci 2007;25:3091-3098.
30 Guo W, Robbins MT, Wei F, Zou S, Dubner R, Ren K: Supraspinal brain-derived neurotrophic factor signaling: a novel mechanism for descending pain facilitation. J Neurosci 2006;26:126-137.

>31 Barreau F, Ferrier L, Fioramonti J, Bueno L: Neonatal maternal deprivation triggers long term alterations in colonic epithelial barrier and mucosal immunity in rats. Gut 2004;53: 501-506.

>32 Stam R, Ekkelenkamp K, Frankhuijzen AC, Bruijnzeel AW, Akkermans LM, Wiegant VM: Long-lasting changes in central nervous system responsivity to colonic distention after stress in rats. Gastroenterology 2002;123:1216-1225.

33 Greisen MH, Altar CA, Bolwig TG, Whitehead R, Wortwein G: Increased adult hippocampal brain-derived neurotrophic factor and normal levels of neurogenesis in maternal separation rats. J Neurosci Res 2005;79: 772-778.

34 Schulte-Herbruggen O, Chourbaji S, Ridder $\mathrm{S}$, Brandwein C, Gass P, Hortnagl H, Hellweg R: Stress-resistant mice overexpressing glucocorticoid receptors display enhanced BDNF in the amygdala and hippocampus with unchanged NGF and serotonergic function. Psychoneuroendocrinology 2006;31: 1266-1277.

35 Aguilar-Valles A, Sanchez E, de Gortari P, Balderas I, Ramirez-Amaya V, BermudezRattoni F, Joseph-Bravo P: Analysis of the stress response in rats trained in the watermaze: differential expression of corticotropin-releasing hormone, CRH-R1, glucocorticoid receptors and brain-derived neurotrophic factor in limbic regions. Neuroendocrinology 2005;82:306-319. 